\title{
Performance and Power Consumption Analysis of IEEE802.11ah for Smart Grid
}

\author{
Zhe Zheng $\mathbb{D}$, Wenpeng Cui, Lei Qiao, and Jinghong Guo \\ Department of Information and Communication, Global Energy Interconnection Research Institute, State Grid Key Laboratory of \\ Information \& Network Security, State Grid Laboratory of Electric Power Communication Network Technology, Beijing, China \\ Correspondence should be addressed to Zhe Zheng; zhengzhe@geiri.sgcc.com.cn
}

Received 19 April 2018; Revised 27 June 2018; Accepted 9 July 2018; Published 25 July 2018

Academic Editor: Fuhui Zhou

Copyright (C) 2018 Zhe Zheng et al. This is an open access article distributed under the Creative Commons Attribution License, which permits unrestricted use, distribution, and reproduction in any medium, provided the original work is properly cited.

\begin{abstract}
IEEE802.11ah is a Wireless Local Area Network (WLAN) designed for the application of Internet of Things (IoT) and Machine to Machine (M2M), mainly used in sensor network, smart metering, car network, health care, and other emerging fields. IEEE802.11ah inherits the IEEE802.11n \ac technology. At present, smart grid has completed the installation of optical fiber communication as its backbone network; WLAN can be used to build new wireless sensor network for smart grid by improving the transmission distance, speed, and power efficiency. The critical features of 802.11ah make it a powerful candidate for WLAN in smart grid, such as intelligent substation sensor network and automatic metering system (AMS). This paper simulates the new added highly robust $1 \mathrm{MHz}$ bandwidth and Modulation Coding Scheme (MCS) 10 in the 802.11ah physical layer and analyzes the coverage range and energy-saving performance of 802.11ah based on the simulation results. The analysis shows that the 802.11ah at $1 \mathrm{MHZ}$ MCS 10 mode can obtain 2.5-3 dB gain. Combined with advantages of Sub-1GHz working frequency, 802.11ah could enlarge the coverage range by five times, compared to $2.4 \mathrm{GHz}$ Wi-Fi. On the other side, 802.11ah module used in the smart grid can greatly reduce power consumption, especially in the AMS with a $1.1 \mathrm{~kW}$.H power savings per year. 802.1lah not only provides the flexibility and low cost features of wireless communication, but also brings coverage and energy-saving performance improvements, which leads to good economic benefit.
\end{abstract}

\section{Introduction}

IEEE 802.11ah is a wireless networking protocol published in 2017 called Wi-Fi HaLow as an amendment of the IEEE 802.11-2007 wireless networking standard [1]. It operates at the Sub-1GHz (S1G) industrial, scientific, medical (ISM) radio-band [2]. Due to the lower center frequencies, more robust PHY layer, and enhancement on MAC layer protocol, the transmission range is extended to $1 \mathrm{~km}$, the data rates are at least $100 \mathrm{kbps}$, and the number of nodes permitted is raised up to $8191[1,3]$. Benefiting from the lower power consumption, it can support the application of the Internet of Things (IoT) by creating large groups of STAs and sensors that cooperate to share signals [4]. It aims at providing connectivity to thousands of devices under one access point (AP).

Due to the advantages of wide coverage, flexible access, low investment, and maintenance costs, as well as good scalability [5], wireless communication has been widely used for many years. However, traditional grid does not prefer the wireless communication for its weakness of reliability. As the development of smart grid, demands for sensor network have been raised to support massive data collection for smart decisions. Therefore, combined with its advantages and improvement on reliability, wireless communication should have a priority for building new network for smart grid.

The networks in substation and automatic metering system (AMS) are most typical in smart grid. WLAN is suitable for the two scenarios by providing the connection between the sensor and the access point in the control room or between the meter and the concentrator. Because smart grid needs to realize better single hop coverage and faster reaction than the other IoT application, the WLAN should improve its transmission distance and speed in order to be admitted [6]. 802.11ah, which claims to extend the coverage to $1 \mathrm{~km}$ and provide at least $150 \mathrm{kbps}$ data rate while maintaining a low power consumption, could be a competitive candidate for the new wireless communication of smart grid [7]. 
To discuss the feasibility of 802.11ah protocol used as a communication standard for smart grid, the data transmission features of the smart grid should be analyzed and compared to the specifications of 802.11ah. In the investigation, a simulation of the 802.11ah physical layer and a derivation of coverage range had been done to prove the feasibility. From the simulation results and analysis, 802.1lah can cover larger area than other 802.11 protocols while still maintaining a enough data rate. A further power consumption analysis was accomplished to prove economic benefit by adopting 802.11ah in smart grid.

\section{Improvements on Physical Layer and Power Consumption}

2.1. Physical Layer. In the physical layer, 802.11ah operates at the S1G. It not only supports the $2 \mathrm{MHz}, 4 \mathrm{MHz}, 8 \mathrm{MHz}$, and $16 \mathrm{MHz}$ bandwidth transmission mode, but also adds $1 \mathrm{MHz}$ bandwidth with MCS0-rep2 to achieve $>1 \mathrm{~km}$ coverage range [1]. It also supports relaying to obtain further improvement on the coverage range [8]. Directional antenna is also supported to solve the hidden terminal problem.

The target spectrum of 802.11ah is as follows: 863868.6 MHz (Europe), 950.8 MHz-957.6 MHz (Japan), 755$787 \mathrm{MHz}$ (China), 917-923.5 MHz (Korea), and 902-928 MHz (USA) [1]. For example, the spectrum in China is shown in Figure 1. 802.11ah adopts OFDM to improve the transmission data rate. The subcarriers could be divided into $1 \mathrm{MHz}$ and $2 \mathrm{MHz}$ and above bandwidth system. For $2 \mathrm{MHz}$ and above bandwidth, the distribution of subcarriers follows the rules of $802.11 \mathrm{ac}$ with one-tenth of the frequency. For $1 \mathrm{MHz}$ bandwidth, 802.11ah utilizes 32 subcarriers and 32-point IFFT. It includes 1 DC component, 2 pilot components, 24 data carriers, and 5 zero components [3].

The subcarrier spacing $\Delta f$ is $31.25 \mathrm{kHz}$, one-tenth of 802.11ac. The guard interval (GI) duration TGI is 8us and 4 us for short GI, respectively. The OFDM symbol duration $T_{S Y M L}$ with long GI is 40 us and 36 us for short GI, respectively.

The modulation and coding scheme (MCS) of 802.11ah are presented in Table 1. Differing from 802.11ac, a robust mode MCS10 has been added.

2.2. Power Optimization Technology. The IEEE802.11ah standard defines two power consumption management modes, namely, activation mode and low power consumption mode. In the activation mode, the STA keeps the RF module in an open state, and the data can be continuously transmitted when there are upstream and downstream data operations. On the other hand, in low power mode, STA switches between hibernation and activation [2]. When hibernating, the STA completely shuts down the RF module and stops receiving signal; in this case, even if the AP has downstream data transmission to the STA, the data can only be cached by AP. When the STA wakes up, switches to the active mode, and sends the downstreaming request, the AP can send the cached data to the STA.

In the IEEE802.11 system, AP periodically broadcasts Beacon frames, and STA periodically examines the TIM IE in

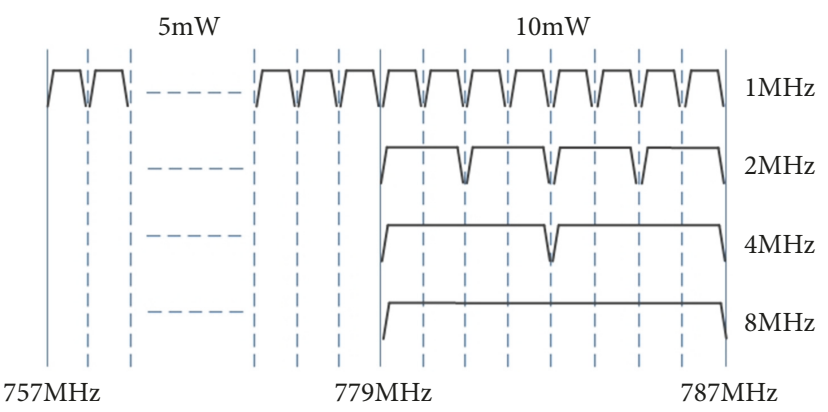

FIGURE 1: 802.11ah frequency band and transmit power limits.

TABLE 1: Modulation and coding schemes.

\begin{tabular}{lccc}
\hline MCS & Mod & Rate & Data-Rate (kbps) \\
\hline 0 & BPSK & $1 / 2$ & 300 \\
\hline 1 & QPSK & $1 / 2$ & 600 \\
\hline 2 & QPSK & $3 / 4$ & 900 \\
\hline 4 & $16-Q A M$ & $1 / 2$ & 1200 \\
\hline 5 & $16-Q A M$ & $3 / 4$ & 1800 \\
\hline 6 & 64-QAM & $2 / 3$ & 2400 \\
\hline 7 & 64-QAM & $3 / 4$ & 2700 \\
\hline 8 & 64-QAM & $5 / 6$ & 3000 \\
\hline 9 & 256-QAM & $3 / 4$ & 3600 \\
\hline 10 & 256-QAM & $5 / 6$ & 4000 \\
\hline
\end{tabular}

Beacon to check if there is data to be received. If so, a PS-Poll must be sent to request the AP to start a packet transmission. After completing the transmission, STA goes to sleep mode.

However, under this mechanism, problems arise when there are a large number of STAs in the network. The TIM IE Partial Virtual Bitmap of Beacon frames will become very long. If overmuch packets are cached in AP, many low power STA may not be able to complete the data transmission in a Beacon interval and always maintain activation patterns, which causes huge power consumption.

In order to solve this problem, IEEE802.11ah standard puts forward a new traffic information indicator (TIM) diagram and the Page Segmentation strategy, the AP change the virtual bitmap from one page into multiple pages, and each Beacon carries their corresponding Page Segmentation STA. A new Segment Count IE is introduced, containing the splitting distribution and boundary information for each page segment.

DTIM Beacon is a new Beacon frame that contains the new TIM IE for grouping processing packets. When DTIM Beacon is broadcasted or multibroadcasted, all Group STA need to be woken up to see if there is cache data in the group. If the entire group does not have cached data, the whole group stays asleep throughout the DTIM cycle until the next TIM Beacon arrives. DTIM cycle is an integer multiple of TIM, giving STA more time to sleep, resulting in a significant reduction on power consumption. 
In addition, 802.11ah introduces two other optimization strategies [2].

Maximum Sleep Time Expansion: use the maximum two bits of the maximum idle cycle field as a scaling factor $.00,01,10,11$, which represents the scale factor of $1,10,1000$ and 10000. The maximum sleep time can be extended up to 2500 times. Second, allow the AP to set the maximum free period for different values at different STAs, and the STA can request the allocation of specific values in the associated request.

Target Wakeup Time (TWT): TWT mechanism allows AP to arrange STA wakeup time, which makes different stations wake up at different time, thereby reducing competition and collision, improving the system efficiency and also greatly reducing the power consumption of the STA. Each time the STA wakes up for several TWT Service Periods (SP), the station sends data through the traditional channel access mode during the TWT SP. STA can stay awake outside of TWT SP time slots, and may even receive the Beacon frame when sleeping. 802.11ah also proposes TWT grouping mechanism, AP builds TWT group, and notifies STA grouping information.

\section{Demands of Smart Grid}

As the development of smart grid, the communication network has been getting increasing attention. Compared with the wired communication technology, wireless communication technology has the advantages on mobility, simplicity, and flexibility of installation, cost reduction, and expandability.

The emerging $\mathrm{M} 2 \mathrm{M}$ wireless communication standards, IEEE 802.11ah, are expected to be used in intelligent substation sensor network and AMS, which is the most common WLAN in smart grid.

3.1. Sensor Network in Substation. Online monitoring is one of the most important applications in power system. As the development of the online monitoring technology, it has been widely admitted and adopted in condition overhaul in the substation $[5,9]$.

The online monitoring system must include large number of diverse sensors, like temperature sensor, humidity sensor, CT sensor, PT sensor, vibration sensor, stress sensor, etc. 802.11ah, with the characteristics of long coverage range, low power consumption, large number of access nodes, and fairly high transmission data rate, is very suitable for the communication network of online monitoring in substation to form a connection among the sensor nodes. $1 \mathrm{~km}$ communication distance guarantees the whole coverage for one substation. $8000+$ number of stations guarantees that the capacity for new sensors is plenty. Low power consumption provides the possibility of ten years' duration with small battery. High data rate provides capability for some light video stream nodes. With some modified in the MAC layer, 802.11ah will meet the real-time requirements for industrial application.

3.2. Automatic Metering System. Traditional power meter reading is simple due to the small amount of transmission data. The requirement for real-time transmission is not urgent [10].

The development of smart grid requires real-time mutual interactions between grid and users. Grid needs more data from users to regulate the generation and consumption. Therefore, a new communication system for the automatic metering system, with higher transmission speed, real-time performance, and reliability, should be deployed. For example, traditional AMS only needs to update the user data once in a day, but future AMS needs to update once in 5 minutes. Only in this way is the gird able to realize new function, such as demand response, relying on the real-time data collected.

Now in China, Broadband Power Line Communication (PLC) is being rapidly applied. Compared to the narrowband PLC, Broadband PLC has raised the transmission data rate from $5 \mathrm{kbps}-50 \mathrm{kbps}$ [11] to $100 \mathrm{kbps}-2 \mathrm{Mbps}$, which is a great promotion. But similar to narrowband PLC, broadband PLC malfunctions when noise or impedance mismatch on power lines becomes too big, which always happens when power electronic devices, such as electronic vehicles, are connected to grid. Thus, wireless communication could act as a backup to avoid problems above. The present main wireless communication protocol in China is micropower wireless communication, which only supports a $1-20 \mathrm{kbps}$ transmission data rate. 802.11ah supports $150 \mathrm{kbps}$ at least when using the most robust mode by adopting OFDM and repetition technology. It also improves the real-time performance and reliability at the same time. These features of 802.11ah provides great potential for using it in the AMS communication of smart grid.

\section{Simulation of PHY Layer}

According to the analysis above, for smart grid application, the transmission data rate of $802.11 \mathrm{ah} 1 \mathrm{MHz}$ mode meets the demands for most of the sensor networks and AMS. And the robustness provided by the $1 \mathrm{MHz}$ MCS10 could also promote the reliability of grid communication system. Therefore, $1 \mathrm{MHz}$ mode is simulated in this paper.

This paper use Simulink ${ }^{\circledR}$ tools to simulate the physical layer of $802.11 \mathrm{ah} 1 \mathrm{MHz}$ mode. The communication system toolbox in the Simulink has also been used.

4.1. Structure of PHY Layer Simulation. The structure of 802.11ah physical layer $[1,3]$ is as in Figure 2, which includes blocks as PHY service data unit (PSDU) generator, MCS setting, transmitter, receiver, error rate calculation (ERC), and signal to noise (SNR) calculation.

PSDU generator block uses a random integer generator to simulate the data from the MAC layer. MCS setting block controls the modulation scheme. According to the MCS chosen, transmitter block processes the data and passes it to channel model block which adds the channel affection to the signal; receiver block processes the data from the channel model block in a reverse flow. ERC block at the end of the data link provides the bit error rate (BER) by delaying the original data from the PSDU block and comparing it to the output data of the receiver block. SNR estimation block calculates 


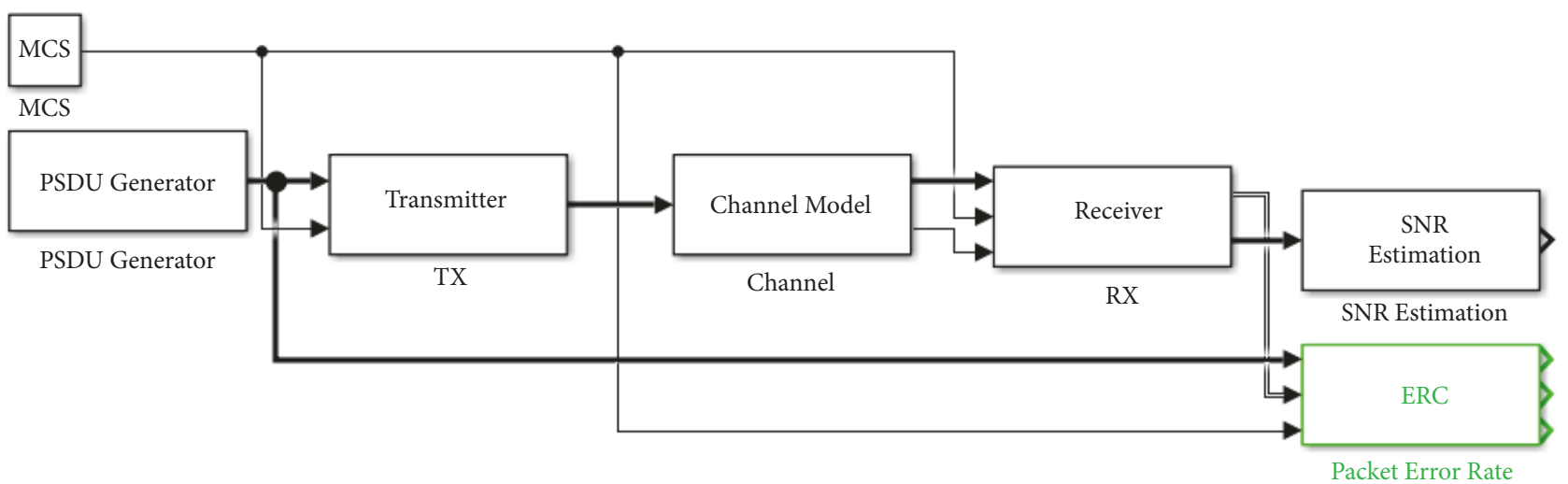

Figure 2: Structure of whole PHY layer simulation.

the SNR from the final signal. The transmitter and receiver are the critical blocks of the whole module.

The structure of the transmitter block is shown in Figure 3. It includes modulation, pilots inserting, zeros adding, IFFT, and cyclic prefix appending blocks. The data stream follows the flow of the 802.11ah standards.

The structure of the receiver block is shown in Figure 4. It includes time domain synchronizing, FFT, frequency domain synchronizing, and demodulation blocks.

4.2. Structure of Repetition Module in MCS 10. The IEEE802.11ah provides two transmitter models. One is the regular nonrepetition MCSs, which is consistent with the IEEE802.11ac transmitter [1].

The second type of transmitter model is MCS0-rep2 model, named MCS $10[1,10]$. As the new scheme proposed, the purpose of this model is to improve the coverage of both indoor and outdoor scenes by using repetition technology when guaranteeing a $150 \mathrm{kbps}$ transmission speed.

The most important feature of MCS 10 is the repetition mechanism adopted in the 24 data subcarriers. The realization by the transmitter is to place the copy of the front 12 bits to the 12 bits behind. On the receiver side, a comparison between upper 12 bits and down 12 bits is made, in order to further improve the transmission reliability.

According to the standard definition, the transmitter of MCS 10 inserts the repetition module after the encoder module and before the interleaver module, as shown in Figure 5. Formula (1) shows the logic operation.

$$
R_{24}=\left\{R_{12}, \operatorname{xor}\left(R_{12},[1,0,0,0,0,1,0,1,0,1,1,1]\right)\right\} .
$$

The receiver of MCS 10 receiver inserts the derepetition module after the deinterlever module and before the Viterbi decoder module, as shown in the Figure 6. Formula (2) shows the logic operation.

$$
R_{12}=\frac{R_{u 12}+\left(\left|R_{d 12}\right| \times\left\{\operatorname{xor}\left[\left(\operatorname{sign}\left(R_{d 12}\right)+1\right) / 2\right],[1,0,0,0,0,1,0,1,0,1,1,1]\right\}\right)}{2}
$$

4.3. Simulation Methods. In smart grid, the reliability of the communication has higher priority than the transmission data rate; therefore 16-QAM, QPSK, BPSK, and $1 \mathrm{MHz}$ bandwidth will be enough in most of the applications. This paper simulates the performance of the physical layer in the case of $1 \mathrm{MHz}$ bandwidth and MCS $0,1,2,3,4,10$. The parameters of the simulation are listed in Table 2.

In the simulation, the Channel Model is AWGN with multipath, SNR is from -5 to 20 with a step of 0.5 , code/decode adopts BCC/Turbo, synchronization and frequency offset correction are assumed to be ideal, data length is 1440 which is 20 symbols, and antenna mode chooses SISO.

A Matlab .m script runs the simulation, records all the BER results under different conditions, and plots the results in one figure for analysis.

\section{Results and Analysis}

5.1. BER Statistics. The BER statistics versus the SNR for different MCS and $1 \mathrm{MHz}$ bandwidth are shown in Figure 7. It is clear that the tolerance to SNR goes lower when more complicated modulation scheme is chosen.

When MCS is 4, modulation is 16-QAM, code rate is $3 / 4$, and the BER reaches $10-1$ when SNR equals $13.5 \mathrm{~dB}$. In most applications of smart grid, the SNR cannot reach $15 \mathrm{~dB}$; therefore it is concluded that MCS 4,5,6,7,8,9 are not suitable for the smart grid for lack of tolerance to SNR.

According to the trend of the data, when SNR is good enough, the BER for different MCSs is too small to tell a performance difference. But when the SNR becomes lower, the effects could be discovered. There is about $3 \mathrm{~dB}$ improvement when MCS moves one step in the robust direction. 


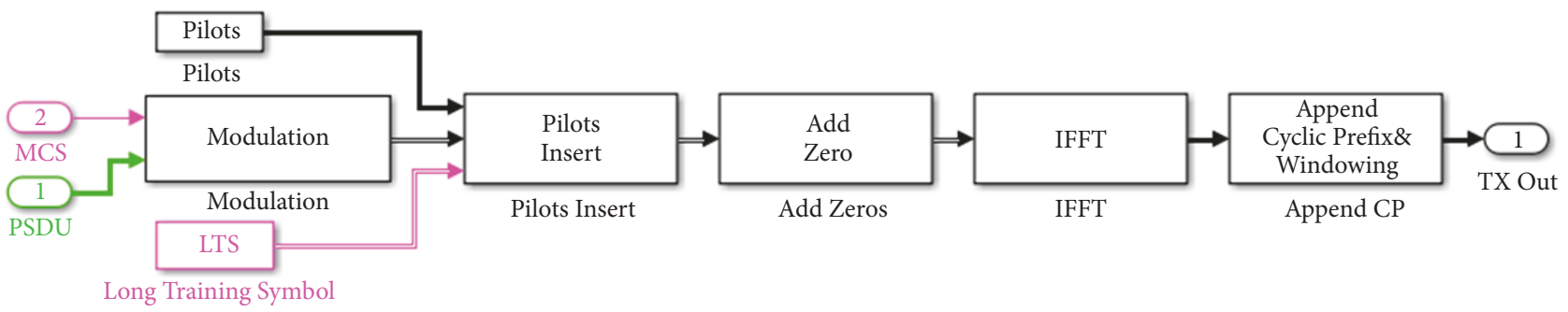

FIgURE 3: Structure of PHY layer transmitter.

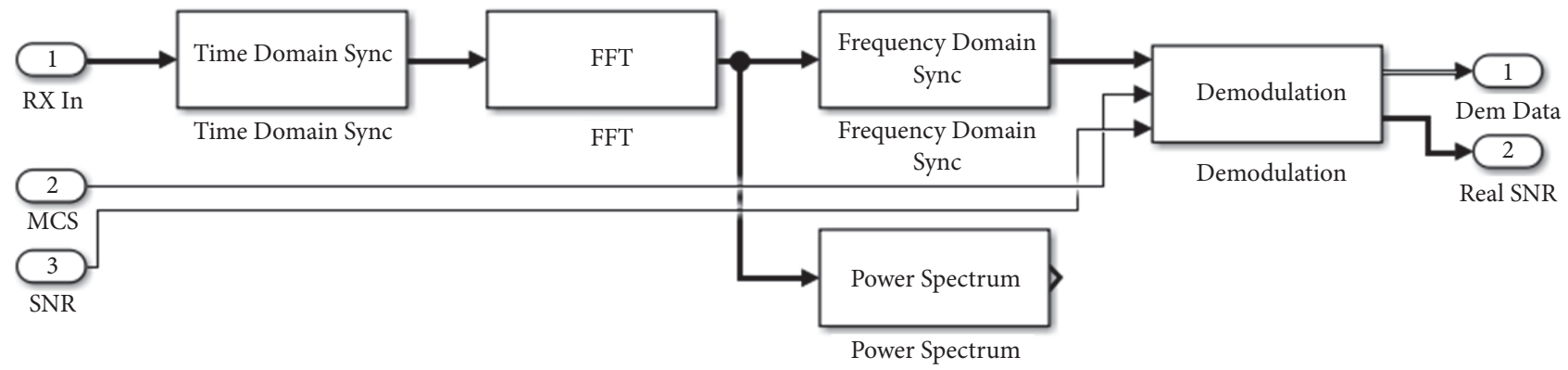

FIGURE 4: Structure of PHY layer receiver.

According to experience, BER equals $20 \%$ is set to the limit of malfunctions, and the SNR can reach a practical value of $10 \mathrm{~dB}$ in smart grid. When MCS equals 0,1,2, and 10, it satisfied the requirements. According to Table 1, 802.11ah could provide transmission capability of $150 \mathrm{kbps}, 300 \mathrm{kbps}$, $600 \mathrm{kbps}$, and $900 \mathrm{kbps}$. This conclusion of transmission data rate is similar to the result of broadband PLC field test.

In the scheme of MCS 0 , the most robust scheme without repetition, the BER will reach the limit when $S N R$ reduces to 0 . This paper also simulates the scheme of MCS 10, which applies the repetition. From result shown in Figure 4, a 2.5$3 \mathrm{~dB}$ improvement has been found compared to MCS 0 when the SNR is smaller than 0 . When the SNR restores to $2-2.5 \mathrm{~dB}$, there is no difference between MCS 10 and MCS 0 .

5.2. Coverage Analysis. In the 802.11 ah communication system, link budget can be expressed with formula $(3)[4,7]$, where $P_{R X}$ is the receiving power, $P_{T X}$ is the transmitting power, $G_{R X}$ and $G_{T X}$ are the antenna gain of receiver and transmitter, $L_{R X}$ is the receiver system loss, $L_{T X}$ is the transmitter system loss, and $P L(f, d)$ is the path loss.

$$
P_{R X}=P_{T X}+G_{T X}-L_{T X}-P L(f, d)+G_{R X}-L_{R X} .
$$

If we neglect system losses $L_{R X}$ and $L_{T X}$ and take fade margins, SNR, and thermal noise power density into account, formula (4) is obtained $[4,7]$.

$$
\begin{aligned}
R_{d B}(f, d)= & P_{T X}+G_{T X}-P L(f, d)-F M(d)+G_{R X} \\
& -\left(\frac{S}{N}\right)_{d B}+N_{o} .
\end{aligned}
$$

TABLE 2: Parameters of simulation.

\begin{tabular}{lc}
\hline Simulation & Parameter \\
\hline MCS & $0,1,2,3,4,10$ \\
\hline Channel & AWGN+Multipath \\
\hline SNR & $(-5,0.5,20)$ \\
\hline Code/Decode & BCC/Turbo \\
\hline Bandwidth & $1 \mathrm{MHz}$ \\
\hline Synchronization & Ideal \\
\hline Frequency Offset Correction & Ideal \\
\hline Data Length & 1440 \\
\hline Antenna & SISO \\
\hline
\end{tabular}

The SNR can be expressed as

$$
\left(\frac{S}{N}\right)_{d B}=\left(\frac{E_{b}}{N_{o}}\right)_{d B}+10 \log _{10}\left(\frac{R}{B}\right) .
$$

By substituting formula (5) into formula (4), we have

$$
\begin{aligned}
R_{d B}(f, d)= & P_{T X}+G_{T X}-P L(f, d)-F M(d)+G_{R X} \\
& -\left(\frac{E_{b}}{N_{o}}\right)_{d B}-10 \log _{10}\left(\frac{R}{B}\right)+N_{o} .
\end{aligned}
$$

In (6), $E_{b}$ is the required energy for each information bit transmission, $N_{o}$ is the thermal noise power density, $R$ is bit rate, and $B$ is system bandwidth.

Since there is currently no 802.11ah commercial product for testing, it is practical to estimate its coverage performance by comparing it with $2.4 \mathrm{GHz} \mathrm{Wi}$-Fi. For example, in China, the transmitting power is $10 \mathrm{~mW}$, and the working frequencies 


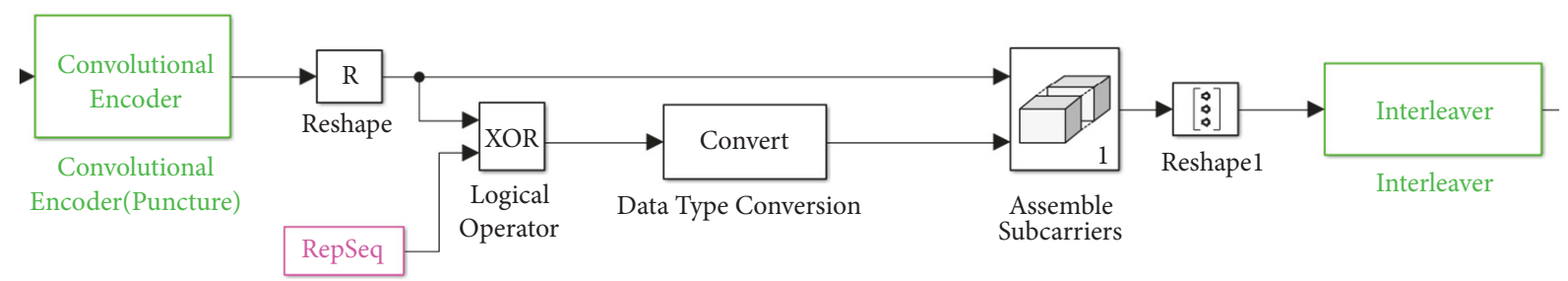

Repetition XOR Sequence

FIGURE 5: Repetition module in transmitter modulation.

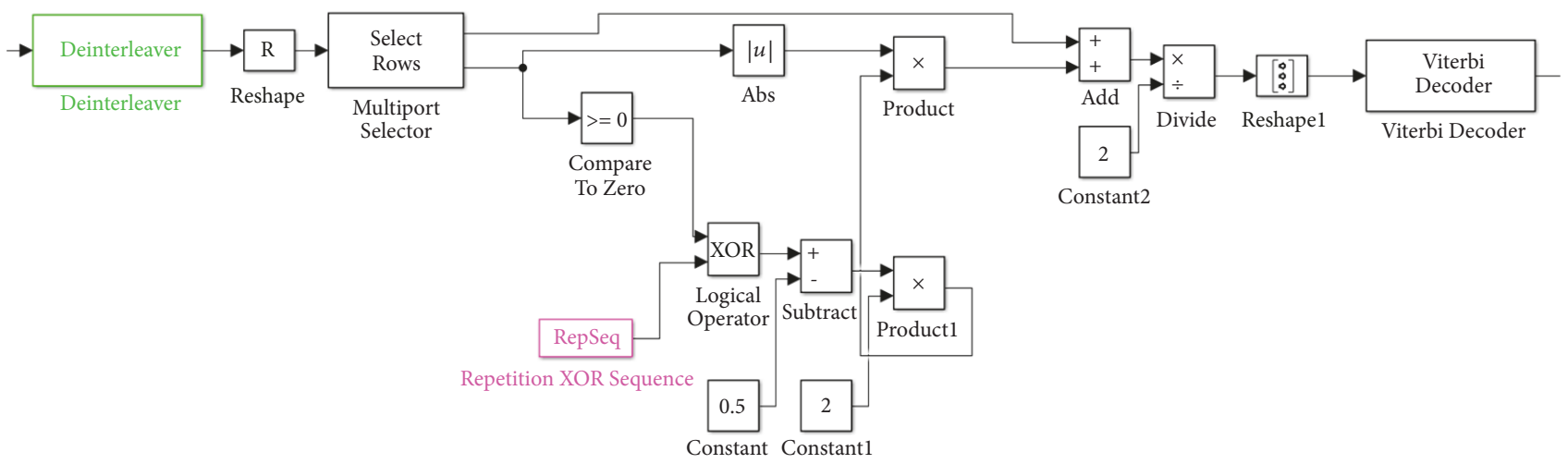

FIGURE 6: Derepetition module in receiver demodulation.

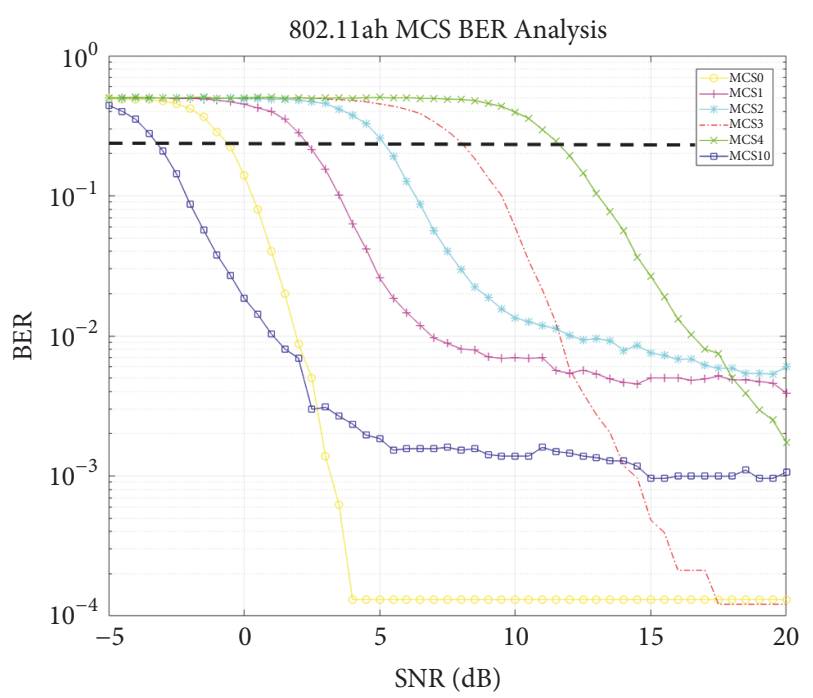

FIGURE 7: BER versus SNR with different MCS.

are $779-787 \mathrm{MHz}$. If using the most robust scheme, MCS 10 , $1 \mathrm{MHz}$ bandwidth and repetition mode are adopted.

$P L(f, d)$ can be expressed by formula (7), in which the $f_{M H z}$ is the working frequency in $\mathrm{MHz}$ and the $d_{k m}$ is the transmission distance in kilometers.

$$
P L(f, d)=32.44+20 \log _{10} f_{M H z}+20 \log _{10} d_{k m} .
$$

TABLE 3: Improvements of 11ah in dBs compared to $2.4 \mathrm{GHz}$ Wi-Fi.

\begin{tabular}{|c|c|c|c|}
\hline \multirow[t]{2}{*}{ Parameter } & \multirow{2}{*}{$\begin{array}{c}\text { Improvement of } \\
780 \mathrm{MHz} 11 \mathrm{ah} \\
\text { Over } 2.4 \mathrm{GHz}\end{array}$} & \multicolumn{2}{|c|}{$\begin{array}{c}\text { Critical Impact } \\
\text { Factor }\end{array}$} \\
\hline & & 11ah & WiFi \\
\hline $\begin{array}{l}\text { Transmit Power } \\
\text { compared to } 17 \mathrm{dBm}\end{array}$ & $-7 \mathrm{~dB}$ & $10 \mathrm{dBm}$ & $17 \mathrm{dBm}$ \\
\hline TX and RX antenna gain & $0 \mathrm{~dB}$ & - & - \\
\hline Free Space Path Loss & $9.8 \mathrm{~dB}$ & $780 \mathrm{MHz}$ & $2.4 \mathrm{GHz}$ \\
\hline $\begin{array}{l}\text { Noise Bandwidth } \\
(1 \mathrm{MHz})\end{array}$ & $13 \mathrm{~dB}$ & $1 \mathrm{MHz}$ & $20 \mathrm{MHz}$ \\
\hline Flat Fading & $-4.5 \mathrm{~dB}$ & $780 \mathrm{MHz}$ & $2.4 \mathrm{GHz}$ \\
\hline $2 \mathrm{x}$ repetition coding & $2.5 \mathrm{~dB}$ & MCS 10 & - \\
\hline Total & $13.8 \mathrm{~dB}$ & - & - \\
\hline
\end{tabular}

Therefore, the corresponding difference value $(\mathrm{dB})$ of each parameter compared with $2.4 \mathrm{GHz}$ Wi-Fi can be expressed as

$$
\begin{aligned}
\Delta P_{T X} & =(10 m W)_{d B m}-17 d B m, \\
\Delta P L(f, d) & =20 \log _{10} f_{M H z, a h}-20 \log _{10} f_{M H z, 2.4 G} \\
\Delta\left(\frac{S}{N}\right)_{d B} & =10 \log _{10}\left(\frac{R}{B_{a h}}\right)-10 \log _{10}\left(\frac{R}{B_{2.4 G}}\right) .
\end{aligned}
$$

With the calculation of the above equations, the results are shown in the Table 3 [12]. The total improvement of 802.11ah compared with $2.4 \mathrm{GHz}$ Wi-Fi is $13.8 \mathrm{~dB}$. Formulas (11), (12), and (13) [4] approximately express the indoor and outdoor 
$P L(d)$. It is concluded that the path loss of both indoor and outdoor situation has an exponential relation to the extreme transmission distance.

$$
\begin{aligned}
& P L(d)=30.29+20 \log _{10} d \\
& P L(d)=L_{F S}=20 \log _{10}\left(\frac{(4 \pi f d f)}{c}\right) \quad \text { for } d \leq d_{B P}, \\
& P L(d)=L_{F S}+3.5 \log _{10}\left(\frac{d}{d_{B P}}\right) \quad \text { for } d>d_{B P},
\end{aligned}
$$

13.8 $\mathrm{dB}$ improvement can be equivalent to about 5 times transmission distance. According to the field test experience of transformer substation, the transmission distance of $2.4 \mathrm{GHz}$ Wi-Fi is about $30 \sim 50 \mathrm{~m}$ indoor and $100 \sim 200 \mathrm{~m}$ outdoor. Therefore, it can be inferred that the transmission distance of 802.11ah is about 150 250 $\mathrm{m}$ indoor, and about $500 \sim 1000 \mathrm{~m}$ outdoor, which is good enough for most of the scenes in smart grid.

5.3. Power Consumption Analysis. In the applications of AMS, in order to realize the demand response, the current metering frequency, one time per day, is required to upgrade to one time per 5 minutes. According to the electric power company estimation, each metering transmission for demands response has a quantity of $20 \mathrm{k}$ bits of data to report. If the transmission rate of $802.11 \mathrm{ah}$ is $100 \mathrm{kbps}$, assuming one concentrator manages up to 300 power meters, a 60second duration, at most, is needed for data transmission, in which the communication module works at maximum power. Hence, a 802.11ah communication STA module will be in sleep mode for $80 \%$, in receiving mode for $19.94 \%$, and transmitting mode for $0.06 \%$ of the 5 -minute period. As shown in the figure, the power consumption budget of the 802.11ah chip designed by the State Grid is shown in Table 4.

The average power consumption of 11ah STA module could be calculated by (14), which is $309.8 \mathrm{~mW} . \mathrm{s}$ in 5 minutes. It equals 0.024 W.h per day.

$$
\begin{aligned}
P_{\text {average }}= & P_{\text {total }} \times T_{\text {tx }}+\left(P_{r x}+P_{M C U}\right) \times T_{r x}+P_{\text {sleep }} \\
& \times T_{\text {sleep }} .
\end{aligned}
$$

Compared to the broadband PLC module with the transmission rate of the same $100 \mathrm{kbps}$. The power of STA module is 3 watts in maximum load. Using equation $(\mathrm{x})$, it consumes 0.528 W.h per day. Using 802.11ah module, each module will significantly save about 0.5 Wh per day. In China, about 600 million smart meters have been installed, which could save up to $300 \mathrm{k}$ kW.h per day, and 1.1 billion kW.h per year.

In the application of intelligent substation sensor network, referring to industrial automation work patterns, 1lah communication module is in sleep mode for $99.8 \%$ of the time. In the remaining $0.2 \%$, receiving takes $95.7 \%$, transmitting takes $0.1 \%$, and idle states takes $4.2 \%$. One $2200 \mathrm{mAh}$ lithium-ion battery can be used for five years [2]. Because the substation will be maintained two times a year, 802.11ah module fully meets the demand as a sensor network node in intelligent substation.
TABLE 4: Estimated power consumption of 1lah chip designed by state grid.

\begin{tabular}{ccc}
\hline & 11ah Components & Power Consumption \\
\hline \multirow{3}{*}{ TX } & TX AFE & $5 \mathrm{~mW}$ \\
& TX Power & $10 \mathrm{~mW}$ \\
& TX Digital & $0.8 \mathrm{~mW}$ \\
\hline \multirow{2}{*}{$\mathrm{RX}$} & RX AFE & $5 \mathrm{~mW}$ \\
& RX Digital & $1.2 \mathrm{~mW}$ \\
\hline \multirow{2}{*}{ MCU(MAC,70 MHz) } & $15 \mathrm{~mW}$ \\
\hline \multicolumn{2}{c}{ Total } & $37 \mathrm{~mW}$ \\
\hline
\end{tabular}

\section{Conclusion}

According to the results and analysis above, it is shown that 802.11ah MCS 10 indeed improves the performance by raising the tolerance to $\mathrm{SNR}$ by $2.5-3 \mathrm{~dB}$, which will benefit the distance and reliability of communication. Furthermore, except MCS 0,1,2,10, MCS 3,4,5,6,7,8,9 are not suitable for harsh communication condition.

When used in smart grid, 802.11ah is able to provide larger coverage range with a radius of up to $1 \mathrm{~km}$ and significant energy-saving results with great economic benefits.

Therefore, 802.11ah standard could be adopted by smart grid communication, but it is suggested to choose the MCS $0,1,2,10$ to achieve compromise between transmission data rate and reliability.

\section{Data Availability}

The authors declare that the data supporting the findings of this study are available within the article.

\section{Conflicts of Interest}

The authors declare that they have no conflicts of interest.

\section{Acknowledgments}

This work has been supported by the project of development on wireless communication chip of electric power system based on 802.11ah under Grant no. SGRIXTMMXS [2015]1128.

\section{References}

[1] IEEE Standards for Information technology-Telecommunications and information exchange between systems Local and metropolitan area networks-Specific requirements Part 11: Wireless LAN Medium Access Control(MAC) and Physical Layer(PHY) Specifications. Amendament 2: Sub $1 \mathrm{GHz}$ License Exempt Operation, IEEE Std 802.11ahTM-2016.

[2] T. Adame, A. Bel, B. Bellalta, J. Barcelo, and M. Oliver, "IEEE 802.11AH: the WiFi approach for M2M communications," IEEE Wireless Communications Magazine, vol. 21, no. 6, pp. 144-152, 2014. 
[3] S. Aust, R. V. Prasad, and I. G. M. M. Niemegeers, "Outdoor Long-Range WLANs: A Lesson for IEEE 802.11ah,” IEEE Communications Surveys \& Tutorials, vol. 17, no. 3, pp. 1761-1775, 2015.

[4] A. Hazmi, J. Rinne, and M. Valkama, "Feasibility study of IEEE 802.11ah radio technology for IoT and M2M use cases," in Proceedings of the 2012 IEEE Globecom Workshops, GC Wkshps 2012, pp. 1687-1692, USA, December 2012.

[5] X. Bao, G. Wang, Z. Hou, M. Xu, L. Peng, and H. Han, "WDM switch technology application in smart substation communication network," in Proceedings of the 2015 5th International Conference on Electric Utility Deregulation and Restructuring and Power Technologies (DRPT), pp. 2373-2376, Changsha, China, November 2015.

[6] Q. Zhang, X. Wang, J. Wang, C. Feng, and L. Liu, "Survey of demand response research in deregulated electricity markets," Dianli Xitong Zidonghua/Automation of Electric Power Systems, vol. 32, no. 3, pp. 97-106, 2008.

[7] S. Aust, R. V. Prasad, and I. G. M. M. Niemegeers, "Performance evaluation of Sub $1 \mathrm{GHz}$ wireless sensor networks for the smart grid," in Proceedings of the 37th Annual IEEE Conference on Local Computer Networks, LCN 2012, pp. 292-295, USA, October 2012.

[8] B. Zhou, H. Hu, S.-Q. Huang, and H.-H. Chen, "Intracluster device-to-device relay algorithm with optimal resource utilization," IEEE Transactions on Vehicular Technology, vol. 62, no. 5, pp. 2315-2326, 2013.

[9] BIAN Baoyin, LI Xiucai, HUANG Xin. Research on wireless communication model and application analysis in substation [J]. Electrotechnical Application, 2015.

[10] S. Aust, R. V. Prasad, and I. G. M. M. Niemegeers, "IEEE 802.11ah: Advantages in standards and further challenges for sub $1 \mathrm{GHz} \mathrm{Wi}-\mathrm{Fi}$," in Proceedings of the 2012 IEEE International Conference on Communications, ICC 2012, pp. 6885-6889, Canada, June 2012.

[11] A. Haidine, B. Adebisi, A. Treytl, H. Pille, B. Honary, and A. Portnoy, "High-speed narrowband PLC in smart grid landscape-state-of-the-art," in Proceedings of the IEEE International Symposium on Power Line Communications and Its Applications (ISPLC '11), pp. 468-473, Udine, Italy, April 2011.

[12] Dr. Eldad Perahia, Enabling Wi-Fi Internet of Things with 802.11ah Technology. Internet of Things Group, July 2015. 


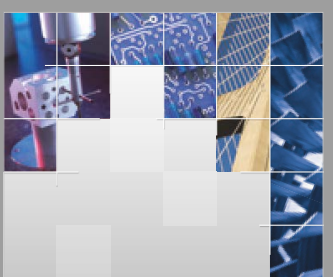

\section{Enfincering}
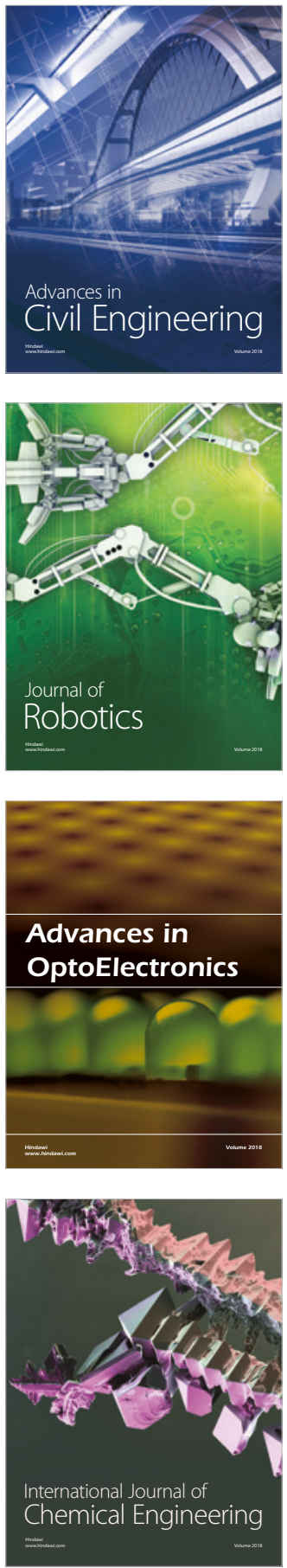

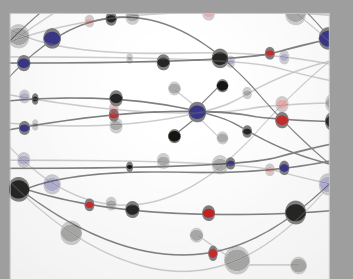

\section{Rotating \\ Machinery}

The Scientific World Journal

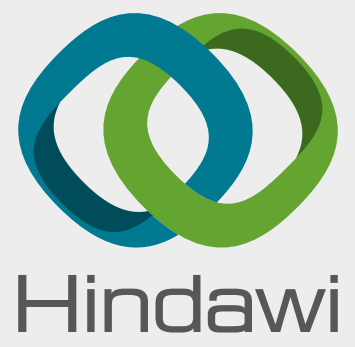

Submit your manuscripts at

www.hindawi.com
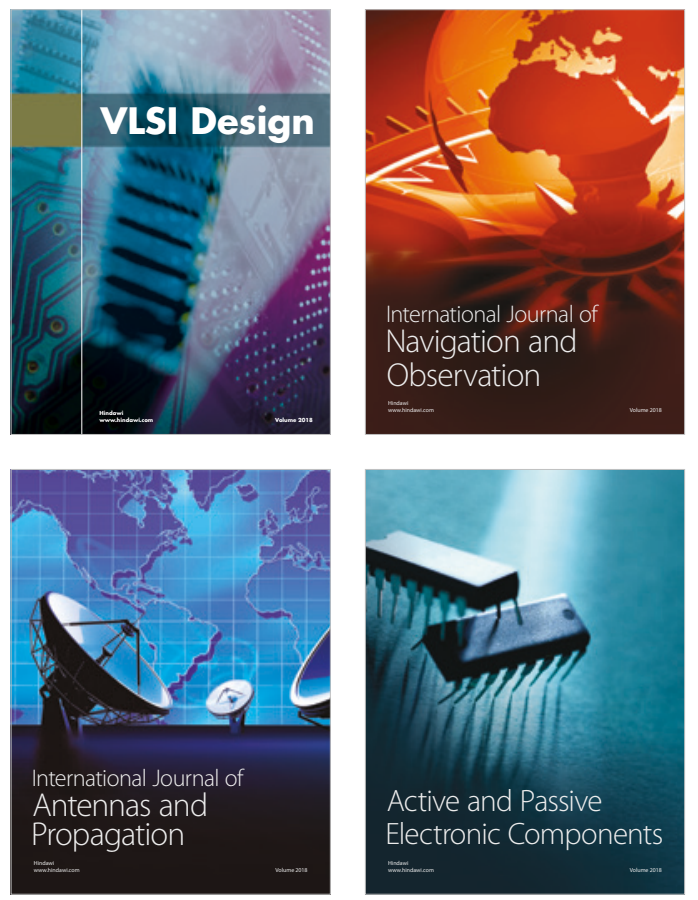
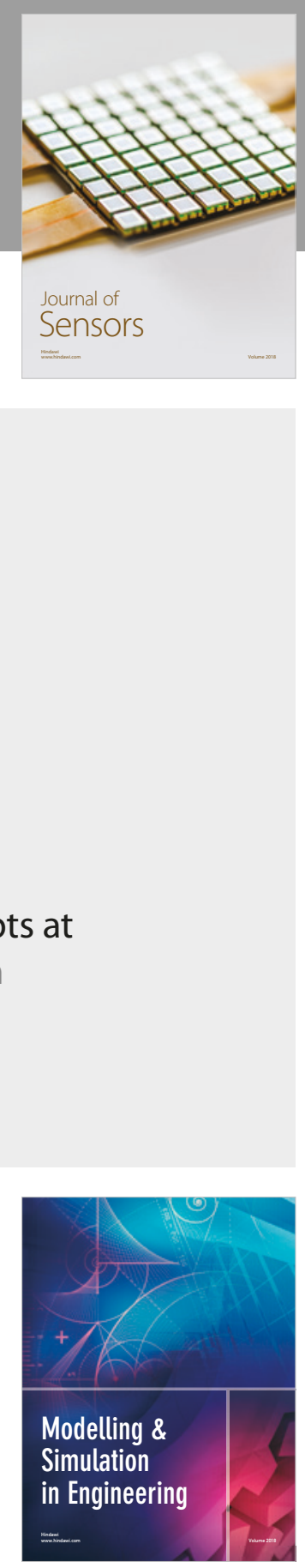

\section{Advances \\ Multimedia}
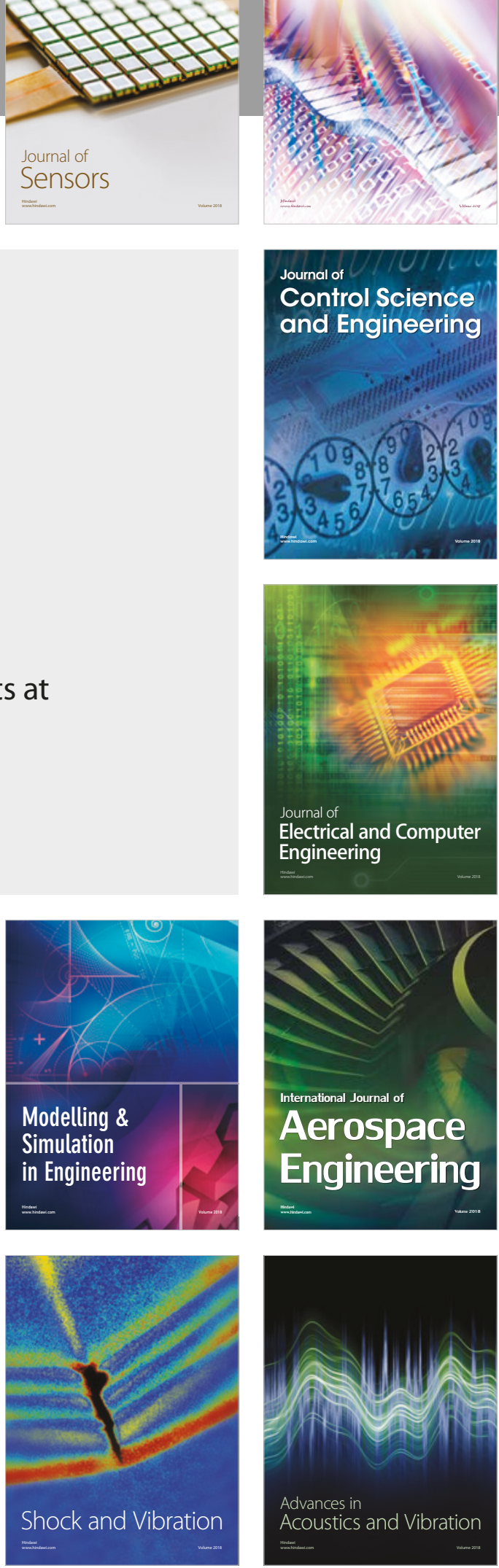